\title{
Modification du microclimat d'un couvert de maïs au moyen de l'irrigation par aspersion en vue de la gestion des stress thermiques des organes reproducteurs
}

\author{
R Saadia ${ }^{1}$, L Huber $^{1 *}$, B Lacroix ${ }^{2}$ \\ 1 Station de bioclimatologie, Inra, F-78850 Thiverval-Grignon; \\ 2 AGPM, 6, chemin de la Côte-Vieille, F-31450 Baziège, France
}

(Reçu le 22 septembre 1995 ; accepté le 10 septembre 1996)

\begin{abstract}
Summary - Using evaporative cooling to fight heat stress in corn. The potential of sprinkler irrigation to reduce air and reproductive organ temperature. During the flowering of corn, heat stress combined with a low water supply may be the cause of unsuccessful fertilization. In order to reduce the heat stress of reproductive organs, evaporative cooling using sprinkler irrigation is a method applicable in seed production. The objective was to characterize the canopy microclimate under irrigation (air temperature at three levels) and quantify the ear temperature reduction as a function of irrigation periodicity and intensity. The experimental study was carried out in the Toulouse region of France on two plots. One plot was irrigated once a week, the other irrigated daily during the warm period of the day (12-17 h GMT). The results showed that ear and tassel temperature decreased immediately after irrigation started, whereas air temperature decreased $2 \mathrm{~h}$ later. Under higher irrigation frequencies, the mean temperature difference between the two plots was about $3{ }^{\circ} \mathrm{C}$ for ears and $2{ }^{\circ} \mathrm{C}$ for air at mid-height inside the canopy; for lower frequencies, the mean temperature difference was $2{ }^{\circ} \mathrm{C}$ for ears and $1{ }^{\circ} \mathrm{C}$ for air inside the canopy. The potential of sprinkler irrigation to reduce heat stress duration on reproductive organs is discussed.
\end{abstract}

evaporative cooling / heat stress / reproductive organ temperature / Zea mays $L=$ corn / sprinkler irrigation

Résumé - La régulation thermique à l'aide de l'irrigation par aspersion aux heures de forte demande climatique est un moyen utilisable pour éviter ou réduire le stress thermique et le stress hydrique qui peuvent provoquer l'échec de la fécondation du maïs. Cette étude a pour objectifs la quantification des modifications thermiques du microclimat du couvert consécutives à l'irrigation par aspersion et l'estimation de la diminution de la température des organes reproducteurs en fonction de la fréquence et de l'intensité d'irrigation. L'irrigation réduit la température des organes reproducteurs immédiatement après le début de l'irrigation alors que la température de l'air subit un abaissement 2 heures plus tard. Pour les fortes fréquences d'irrigation, l'abaissement moyen de la température dans les conditions climatiques rencontrées lors des expérimentations est de $3^{\circ} \mathrm{C}$ pour les épis et de $2^{\circ} \mathrm{C}$ pour l'air à mi-hauteur du couvert. Pour les faibles fréquences d'irrigation, cet abaissement est de $2^{\circ} \mathrm{C}$ pour la température des épis et $1^{\circ} \mathrm{C}$ pour la température de l'air à l'intérieur du couvert. Les potentialités de l'irrigation par aspersion pour la réduction de la durée du stress thermique sont discutées en regard des seuils de température que peut dépasser la température des organes reproducteurs.

refroidissement évaporatif / stress thermique / température des organes reproducteurs / Zea mays $L=$ maïs / irrigation par aspersion 


\section{INTRODUCTION}

Au cours de ces dernières années, on a assisté à des pertes de rendement en production de semences de maïs en liaison avec l'occurrence de fortes chaleurs estivales durant la période de la floraison et de la fécondation. En 1990, la période allant du $1^{\mathrm{er}}$ juillet au 10 août a été une période chaude et sèche marquée par des températures maximales anormalement élevées. Des température supérieures à $35{ }^{\circ} \mathrm{C}$ et des conditions d'hygrométrie situées entre 15 et $20 \%$ ont été enregistrées dans le sud-ouest de la France. Cette période a coïncidé avec la floraison du maïs destiné à la production de semences et provoqué des pertes considérables. Sur l'ensemble du territoire français, $70 \%$ des surfaces ont été touchées dont $30 \%$ ont été irrémédiablement perdues. D'après la Fédération nationale de production de semences de maïs et sorgho, dans la région Midi-Pyrénées où a été menée l'étude expérimentale rapportée dans le présent article, les dégâts causés en 1990 furent estimés à $32 \%$ en pertes de rendement (Lavergne, 1993). Très probablement, les fortes températures et la faible hygrométrie de l'air sont les principales causes de l'échec de la fécondation.

En conditions contrôlées, Herrero et Johnson (1980) ont montré que l'augmentation de la température de l'air de 27 à $38^{\circ} \mathrm{C}$ conduit à une diminution significative du pourcentage de germination du pollen de maïs in vitro. Parmi les 15 génotypes testés par ces auteurs, certains apparaissent plus tolérants au stress thermique que d'autres. Des résultats similaires ont été obtenus par Binelli et al (1985), Forva et al (1986) et Schoper et al (1987b). L'effet de la température se traduit également sur la croissance in vitro du tube pollinique. En exposant du pollen pendant 2 heures à $40^{\circ} \mathrm{C}$, puis en le ramenant à $27^{\circ} \mathrm{C}$, Binelli et al (1985) et Forva et al (1986) ont observé que la croissance du tube pollinique est réduite à $40 \%$ de celle d'un pollen maintenu à $27^{\circ} \mathrm{C}$.

D'autres travaux en conditions contrôlées ont confirmé que les soies sont moins sensibles au stress thermique en conditions de bonne alimentation hydrique et d'hygrométrie élevée (Schoper et al, 1986). Des épillets femelles exposés à $40^{\circ} \mathrm{C}$ pendant 4 heures restent fonctionnels mais le taux de fécondation diminue ; il devient nul après 6 heures d'exposition à cette température (Dupuis et Dumas, 1990). Au cours de la fécondation, une température de $37^{\circ} \mathrm{C}$ pendant
7 jours provoque l'avortement de l'endosperme (Jones et al, 1981) et réduit considérablement le nombre de grains formés (Mitchell et Petolino, 1988).

Certains auteurs ont montré que le stress hydrique a peu d'effet sur la viabilité du pollen de maïs mais réduit la réceptivité des soies. Westgates et Boyer (1986) ont observé que, sous un stress hydrique, la fécondation se fait normalement mais les grains ne se développent pas du fait du blocage de la translocation des assimilats, provoquant l'apparition d'un taux élevé d'anomalies de formation (Moss et Downey, 1971).

Cette différence de sensibilité au stress thermique entre le pollen et les organes femelles s'explique par la synthèse au niveau cellulaire de molécules protectrices. Des protéines de choc thermique ou HSP (Heat shock proteins) sont élaborées par les tissus femelles et leur confèrent une thermotolérance, alors que ces protéines sont absentes chez le pollen mature (Dupuis et Dumas, 1990). Les travaux de Gagliardi (1994) ont montré qu'un stress thermique intervenant 17 à 21 jours avant l'anthèse permet au pollen de synthétiser et accumuler les HSP qui pourraient le protéger pendant la germination et la fécondation.

À partir de ces constatations, des génotypes plus résistants au stress thermique ont été sélectionnés, mais les résultats obtenus furent peu probants : un même génotype peut être résistant aux fortes températures au cours de la formation des organes reproducteurs mais en revanche peut subir des dégâts notables si ces conditions interviennent lors de la floraison-fécondation. C'est sur la base de telles observations qu'il a été suggéré d'intervenir directement au champ, à l'aide de l'irrigation par aspersion pendant les périodes de forte chaleur, pour réduire la température des organes par la mise en jeu du mécanisme de refroidissement évaporatif.

Au champ, l'énergie radiative partiellement transformée en chaleur au niveau des organes végétaux est la première cause du stress thermique qui peut être accentué par l'insuffisance de l'alimentation hydrique. Le principe de base de la régulation thermique est de modifier la répartition des termes du bilan d'énergie par l'introduction d'un terme d'évaporation à la surface des organes végétaux qui va absorber une fraction importante de l'énergie radiative disponible.

Lors de l'irrigation, il y a diminution de la température par évaporation des gouttelettes d'eau pendant leur trajet dans l'air et par évaporation 
de l'eau retenue sur la surface des feuilles (Seginer, 1970; Kohl et Wright, 1974). Le taux de refroidissement par évaporation dépend de la température de surface des organes végétaux, de la température de l'air, du déficit de saturation de l'air et de la vitesse du vent (Griffin et Richardson, 1979 ; Wright et al, 1981). Dans une étude expérimentale qui mettait en jeu l'aspersion d'eau délivrée à $13,5{ }^{\circ} \mathrm{C}$ sous une intensité d'irrigation de $11,6 \mathrm{~mm} /$ heure pendant 3 heures, Thompson et al (1993) ont trouvé que le quart du rayonnement net environ est utilisé pour l'augmentation de la température de l'eau d'irrigation interceptée par les organes végétaux.

Lomas et Mandel (1973) ont observé une diminution de la température de $12{ }^{\circ} \mathrm{C}$ et une augmentation de $27 \%$ de l'humidité de l'air sur une culture d'avocats irriguée par aspersion $4 \mathrm{~m}$ audessus des arbres pendant 1 à 2 heures et $5{ }^{\circ} \mathrm{C}$ et $8 \%$ avec le même système placé à l'intérieur du couvert $(0,25 \mathrm{~m})$. Wright et al (1981) ont pu constater qu'une irrigation de 5 minutes réduit la température des ombelles d'oignon de $15^{\circ} \mathrm{C}$, un refroidissement optimal à l'égard de la fécondation étant obtenu par une irrigation cyclique de 5 minutes toutes les 20 à 30 minutes.

Dans des conditions semi-arides, Kohl et Wright (1974) ont montré expérimentalement que le maximum de refroidissement évaporatif est dû à l'évaporation des surfaces mouillées alors que l'évaporation des gouttes d'eau lors de leur trajet ne provoque qu'une diminution de $1{ }^{\circ} \mathrm{C}$ de la température de l'air. Sur un couvert de maïs irrigué par pivot, Steiner et al (1983) ont trouvé des températures moyennes journalières des feuilles et de l'air inférieures de 2 à $3{ }^{\circ} \mathrm{C}$ par rapport à un couvert soumis à une irrigation au sol.

Le refroidissement par évaporation est donc une méthode de réduction des stress thermiques par réduction de la température des organes végétaux et de l'air, et par diminution de la perte en eau des plantes par transpiration et par augmentation de l'humidité relative de l'air améliorant ainsi les conditions de développement floral et de la fécondation.

Ces résultats sur la protection des cultures contre le stress thermique à l'aide de l'irrigation par aspersion intéressent les producteurs de semences de maïs, ce qui a amené à conduire cette étude dans une des régions fortement touchées par les conséquences du stress. Les objectifs de cette étude concernent : i) la caractérisation de l'incidence de l'irrigation par aspersion sur le microclimat du couvert de maïs lors de fortes demandes climatiques et en particulier la quantification des modifications thermiques; ii) l'estimation de la diminution de la température des organes en fonction de l'intensité et de la fréquence d'irrigation choisies ; iii) l'évaluation de l'efficacité de la régulation thermique pendant les périodes de risque.

\section{MATÉRIELS ET MÉTHODES}

Les essais ont été menés sur le site de la station expérimentale de Baziège (Haute-Garonne, France) sur deux parcelles de $65 \times 60 \mathrm{~m}$ de maïs destiné à la production de semences. Sur chaque parcelle, neuf planches constituées de quatre rangs de plantes femelles (lignée B) et de deux rangs de plantes mâles (lignée F2) ont été semées. L'irrigation par aspersion a été réalisée par un système en couverture intégrale d'asperseurs implantés selon un dispositif en quinconce. La distance entre deux lignes d'asperseurs et entre deux asperseurs sur une ligne était de $18 \mathrm{~m}$. Les asperseurs ont été placés à $2,20 \mathrm{~m}$ de hauteur $(0,5 \mathrm{~m}$ au-dessus du couvert). Deux modalités d'irrigation ont été retenues : i) irrigation hebdomadaire (traitement $\mathrm{H}$ ) pour assurer une bonne alimentation en eau sur la première parcelle qui correspond à un traitement témoin, et ii) irrigation journalière (traitement $\mathrm{J}$ ) pratiquée pendant les heures chaudes de la journée (de 11 ou 12 heures à 17 heures TU).

L'irrigation quotidienne sur la parcelle $(J)$ a été pratiquée par séquence continue de deux minutes d'aspersion appliquée au début de chaque période dont la durée est fixée pour chaque jour. La durée de cette période est de 5 minutes ( 2 minutes d'arrosage et 3 minutes sans aspersion), 10 minutes ( 2 minutes d'arrosage et 8 minutes sans aspersion), 15 minutes (2 minutes d'arrosage et 13 minutes sans aspersion) ou 20 minutes ( 2 minutes d'arosage et 18 minutes sans aspersion). À ces irrigations conditionnées par l'occurrence de fortes chaleurs était ajouté un complément hebdomadaire pour avoir un niveau d'apport hydrique égal au traitement $H$. Pendant la période de mesures, deux intensités d'irrigation ont été testées sur le traitement $(\mathrm{J}): 4,3$ et $7,6 \mathrm{~mm} /$ heure. La température de l'eau d'irrigation se situait généralement entre 20 et $23{ }^{\circ} \mathrm{C}$. Les deux parcelles $(\mathrm{H})$ et $(\mathrm{J})$ étaient juxtaposées et orientées perpendiculairement à la direction des vents dominants dans le but d'éviter l'influence de l'irrigation par aspersion de la parcelle (J) sur le microclimat de la parcelle $(\mathrm{H})$.

Les mesures micrométéorologiques suivantes ont été effectuées sur la parcelle $\mathrm{H}$ : la vitesse du vent à 2,8 et $1,8 \mathrm{~m}$ au-dessus du couvert $(4,5$ et $3,5 \mathrm{~m}$ audessus du sol), au sommet du couvert (1,70 m du sol) et dans le couvert ( $1 \mathrm{~m}$ du sol) avec des anémomètres à coupelles (MCB, Courbevoie, France), la température de l'air aux mêmes niveaux que la vitesse du vent avec des thermocouples (AGW 24) placés dans des abris ventilés, l'humidité relative de l'air au sommet du couvert avec un hygromètre capacitif (Vaisala, Helsinki, Finlande), le rayonnement net au-dessus du 
couvert au moyen d'un pyrradiomètre différentiel (Swissteco, Oberriet, Suisse), la température des épis et panicules par des thermocouples (AGW 30) insérés à $1 \mathrm{~cm}$ sous les spathes de l'épi et dans le brin maître de la panicule. Sur la parcelle J, les mêmes types de capteurs ont été utilisés pour la température de l'air à $3,5 \mathrm{~m}$ au-dessus du sol, au sommet de la culture $(1,70 \mathrm{~m})$ et dans le couvert $(1 \mathrm{~m})$, l'humidité relative de l'air au sommet du couvert, la température des épis et panicules par des thermocouples et la pluviométrie au moyen d'un pluviomètre enregistreur (ARG 100, Campbell Scientific, Shepshed, Royaume-Uni). Les mesures instantanées sont effectuées toutes les 10 secondes et une moyenne calculée toutes les 15 minutes est enregistrée par une centrale d'acquisition pour chaque parcelle (CR10, Campbell Scientific, Shepshed, Royaume-Uni).

Des mesures en conditions standardisées ont été effectuées à une hauteur de $2 \mathrm{~m}$ sur un parc météorologique situé à $300 \mathrm{~m}$ du site expérimental, ces mesures sont : le rayonnement global au moyen d'un pyranomètre (Kipp et Zonen, Delft, Pays-Bas), la température et l'humidité relative de l'air (Rotronic MP100, Campbell Scientific, Royaume-Uni), la vitesse du vent à l'aide d'un anémomètre (MCB, Courbevoie, France) et sa direction avec une girouette (instrument développé par l'Inra). Ces mesures ont été enregistrées à l'aide d'une centrale d'acquisition de données (21X, Campbell Scientific, Shepshed, Royaume-Uni).

\section{RÉSULTATS ET DISCUSSION}

\section{Caractérisation climatique des journées de mesures}

Les expérimentations se sont déroulées entre le 15 juillet et le 25 août 1994, soit une période ayant largement entouré la floraison du maïs qui a eu lieu entre le 27 juillet et le 4 août. Les journées utilisées dans cette étude ont été sélectionnées en fonction de leurs caractéristiques climatiques, à savoir un rayonnement élevé, de fortes températures et de faibles taux d'humidité relative de l'air. Les conditions climatiques rencontrées au champ sont présentées dans le tableau I. On remarque que les conditions de rayonnement et de température de l'air de ces journées sont similaires. À la mi-journée, les valeurs maximales du rayonnement net au-dessus du couvert du site $\mathrm{H}$ (irrigation hebdomadaire) oscillent autour de $600 \mathrm{~W} . \mathrm{m}^{-2}$ et l'énergie moyenne reçue au-dessus du couvert pendant 6 heures (de 11 à 17 heures TU, ce qui correspond à la période d'irrigation du traitement $(\mathrm{J})$ ) est voisine de $10 \mathrm{MJ}^{-2} \mathrm{~m}^{-2}$. La température maximale de

Tableau I. Paramètres climatiques mesurés entre 11 et 17 heures TU au-dessus du site H (irrigation hebdomadaire).

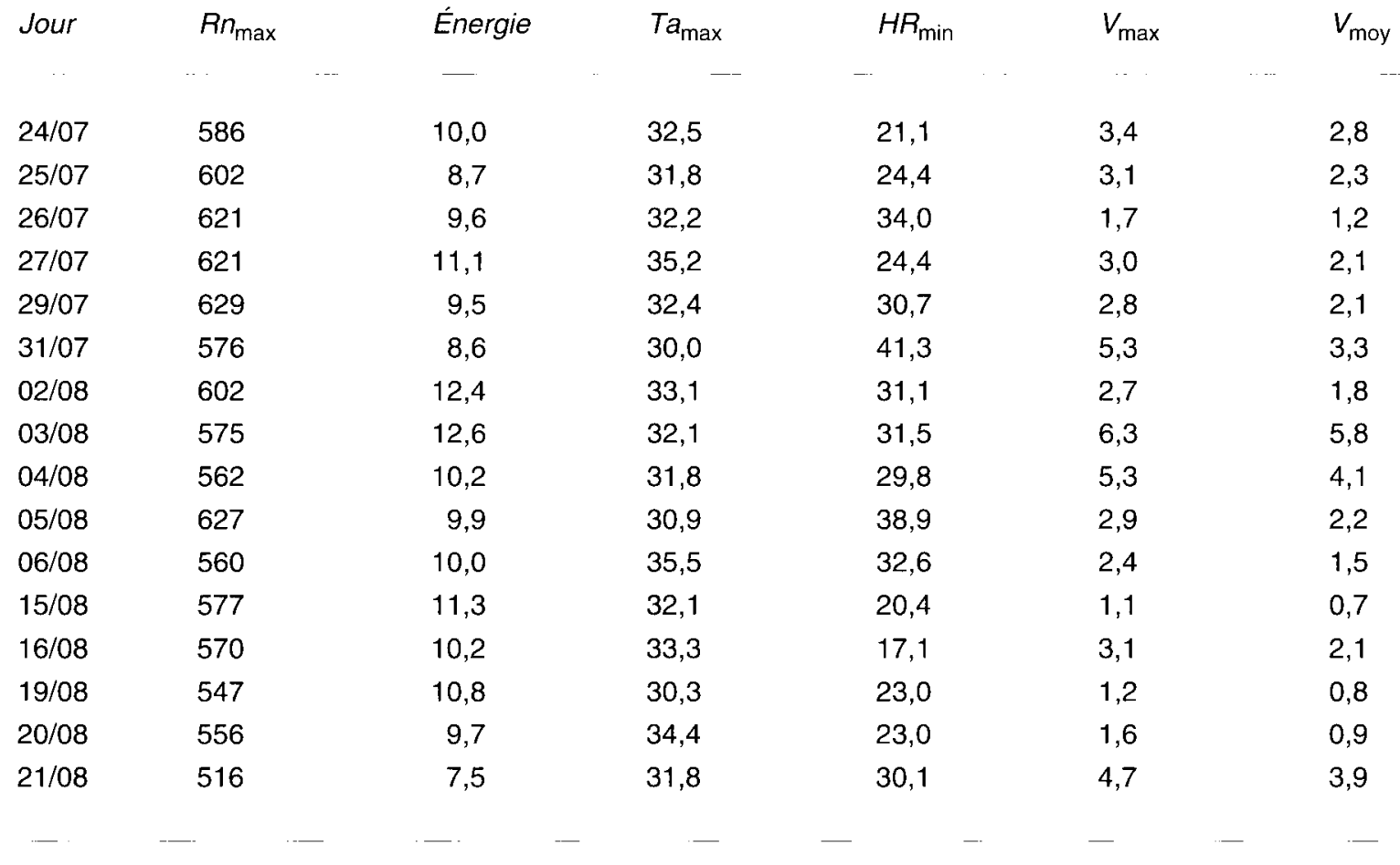

$\mathrm{Rn}_{\text {max }}$ : rayonnement net maximal $\left(\mathrm{W} \cdot \mathrm{m}^{-2}\right)$, énergie moyenne reçu au-dessus du couvert (MJ.m-2), Ta $\mathrm{max}_{\text {max }}$ : température maximale de l'air $\left({ }^{\circ} \mathrm{C}\right)$ mesurée à $4,5 \mathrm{~m}$ au-dessus du sol, $\mathrm{HR}_{\min }$ : humidité minimale de l'air $(\%)$ mesurée au sommet du couvert, $V_{\text {max }}$ et $V_{\text {moy }}$ : maximum et moyenne de la vitesse du vent $\left(\mathrm{m}_{\text {. }} \mathrm{s}^{-1}\right)$ mesurée à $4,5 \mathrm{~m}$ au-dessus du sol. 
l'air mesurée à $4,5 \mathrm{~m}$ au-dessus du sol varie entre 30 et $35^{\circ} \mathrm{C}$. la valeur minimale de l'humidité relative de l'air mesurée au sommet de la culture est très variable (entre 17 et $42 \%$ ).

Variant d'une journée à une autre, la vitesse du vent entre 11 et 17 heures TU (mesurée à une hauteur de $4,5 \mathrm{~m}$ au-dessus du sol) constitue une variable qui intervient dans la régulation thermique, sachant que des valeurs élevées de la vitesse du vent tendent à réduire l'écart entre la température de l'air et celle des organes. On remarque dans le tableau I l'existence de deux catégories de journées correspondant à deux grammes de vitesse de vent : faible (1 à $\left.2 \mathrm{~m} . \mathrm{s}^{-1}\right)$ et moyenne ( 3 à $6 \mathrm{~m} \cdot \mathrm{s}^{-1}$ ). Les journées présentées dans ce tableau étaient très favorables pour mettre en évidence le refroidissement thermique à l'aide de l'irrigation par aspersion. La figure 1 présente l'évolution des variables climatiques mesurées sur le parc météorologique au cours de deux journées (27 et 29 juillet) considérées
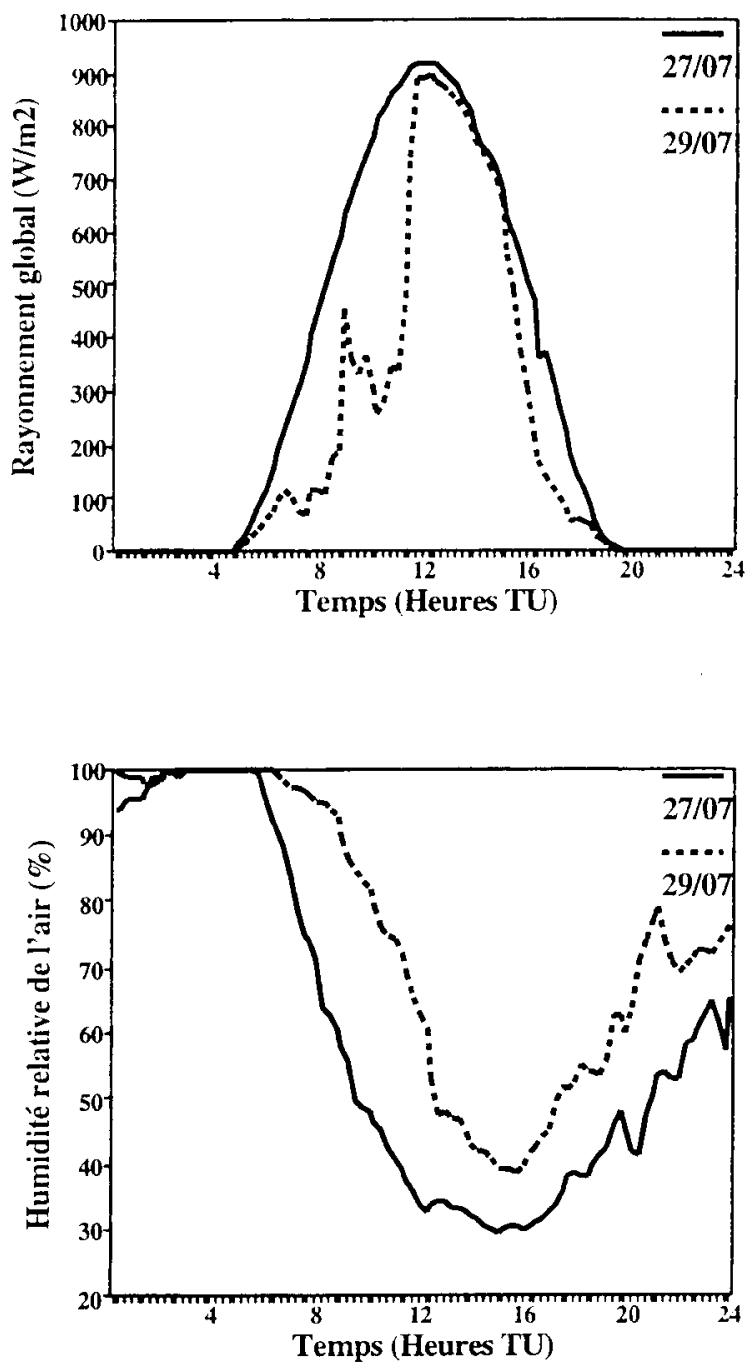

comme typiques, on remarque pour le 27 juillet un fort rayonnement global ( 900 W. $\mathrm{m}^{-2}$ ) autour de 12 heures TU associé à une température supérieure à $30^{\circ} \mathrm{C}$ entre 10 et 19 heures TU. Malgré un rayonnement perturbé le 29 juillet, la température de l'air dépasse $30^{\circ} \mathrm{C}$ entre 11 et 17 heures TU. On remarque également les faibles valeurs minimales de l'humidité relative de l'air ( $30 \%$ le 27 juillet et $40 \%$ le 29 juillet). La vitesse du vent est très faible au cours de la nuit et une partie de la matinée mais elle devient plus élevée au milieu de la journée (gamme de 1 à $4 \mathrm{~m} . \mathrm{s}^{-2}$ ).

\section{Température des organes reproducteurs et température de l'air en l'absence d'irrigation}

La comparaison entre l'évolution de la température des épis et celle de l'air mesurée sur le site irrigué hebdomadairement $(H)$ est présentée sur la figure 2. Au cours de la journée on peut remar-
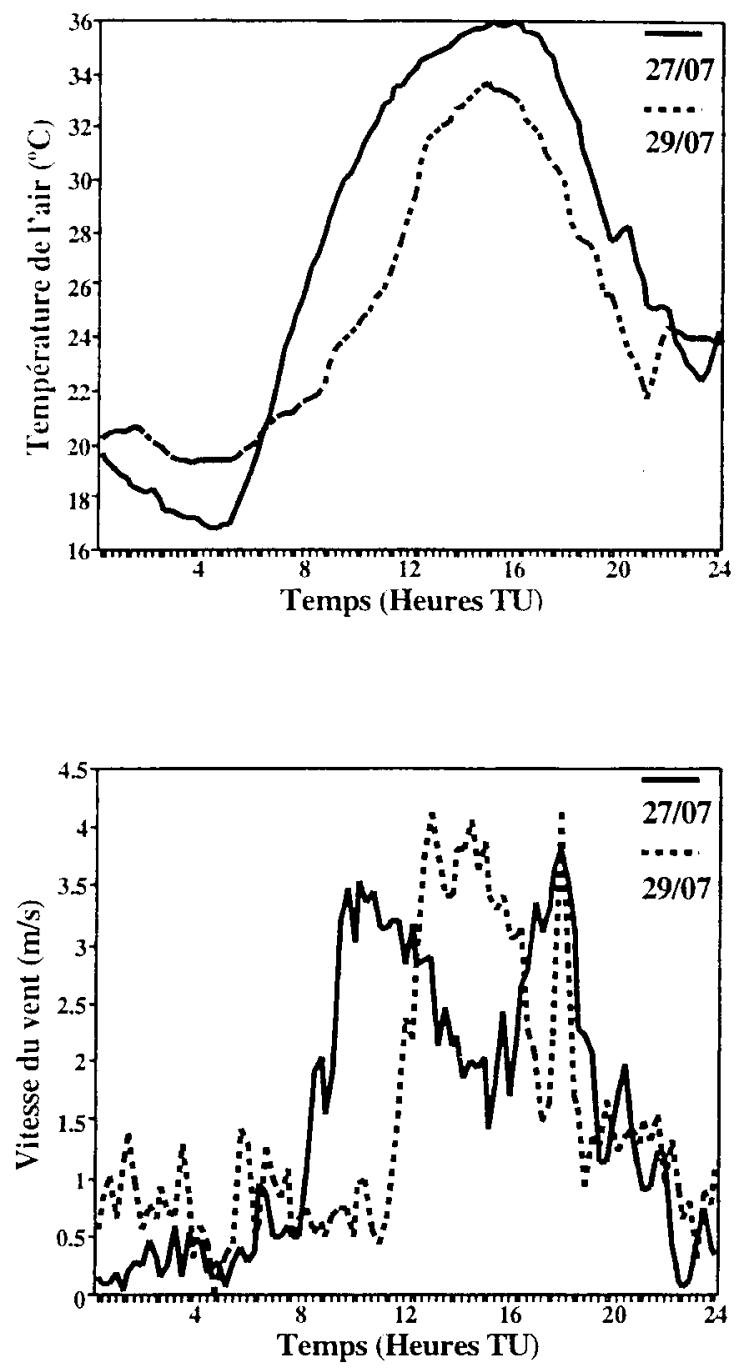

Fig 1. Évolution des variables météorologiques mesurées à $2 \mathrm{~m}$ au-dessus d'une surface engazonnée sur un pas de temps de 15 minutes les 27 (ligne continue) et 29 juillet 1994 (pointillés). 
quer trois phases distinctes. Une phase nocturne où la température des épis est similaire à celle de l'air à mi-hauteur du couvert. Une phase matinale entre 6 et 12 heures TU, où le réchauffement des épis est plus rapide par rapport à l'air, ce qui conduit à une température des épis supérieure à celle de l'air de $1,2^{\circ} \mathrm{C}$ et $3^{\circ} \mathrm{C}$ à midi les jours observés ( 3 et 6 août). Une phase de diminution de la température des épis qui commence vers 12 heures TU et où la température de l'air atteint son maximum $\left(32,5^{\circ} \mathrm{C}\right.$ le 3 août et $35,7^{\circ} \mathrm{C}$ le 6 août). II apparaît un déphasage de 2 heures entre le maximum de la température des épis et le maximum de la température de l'air. L'écart entre les deux maxima est de $1,2^{\circ} \mathrm{C}$ le 3 août et $2,1^{\circ} \mathrm{C}$ le 6 août. De manière générale, la température des épis est inférieure à la température de l'air aux heures critiques de la journée, et ne dépasse pas $33^{\circ} \mathrm{C}$ au cours de la journée la plus chaude rencontrée ( 6 août) alors que la température de l'air mesurée à $4,5 \mathrm{~m}$ audessus du sol sur le traitement $\mathrm{H}$ atteint $35,4^{\circ} \mathrm{C}$.

La différence d'évolution entre la température des épis et la température de l'air (fig 2) s'explique probablement par le réchauffement des épis plus rapide le matin en raison d'une part de leur exposition au rayonnement solaire du fait de
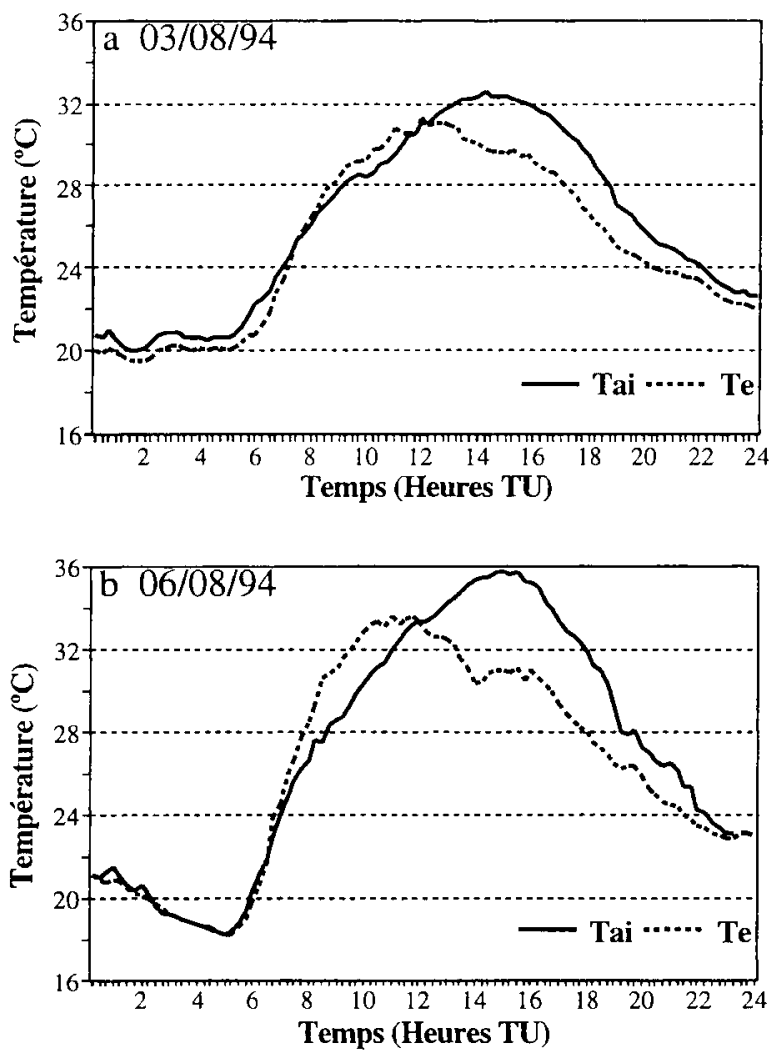

Fig 2. Comparaison entre l'évolution de la température de l'air à mi-hauteur du couvert $(1 \mathrm{~m})$ (Tai) et de la température des épis (Te) sur le site non irrigué $(H)$ le 3 août 1994 (a), le 6 août 1994 (b). la faible densité du couvert (sept plantes par mètre carré) et d'autre part par une faible transpiration due à un taux d'humidité relative de l'air élevé (entre 7 et 12 heures : $52 \%$ le 3 août et $54 \%$ le 6 août) et des vitesses de vent faibles à I'intérieur du couvert (entre 7 et 12 heures : 0,8 $\mathrm{m} . \mathrm{s}^{-1}$ le 3 août et $0,2 \mathrm{~m} \cdot \mathrm{s}^{-1}$ le 6 août). Vers midi la transpiration augmente en réponse à la diminution de l'humidité relative (entre 12 et 17 heures : $37 \%$ le 3 août et $36 \%$ le 6 août) et à l'augmentation de la vitesse du vent (entre 12 et 17 heures : $1,5 \mathrm{~m} . \mathrm{s}^{-1}$ le 3 août et $0,7 \mathrm{~m} . \mathrm{s}^{-1}$ le 6 août) provoquant un refroidissement des tissus.

L'évolution de la température des panicules est similaire à l'évolution de la température de l'air mesurée au sommet du couvert (fig 3) ; les écarts entre les deux températures sont faibles et peuvent atteindre $+2^{\circ} \mathrm{C}$ pendant la matinée. La panicule située au sommet du couvert est donc soumise au rayonnement solaire direct, et bénéficie des conditions de vitesse de vent plus élevées qu'à l'intérieur du couvert. La panicule a une forme aplatie et une structure très dissociée qui favorisent les échanges thermiques; ces caractéristiques contribuent à rapprocher la température de la panicule de celle de l'air.
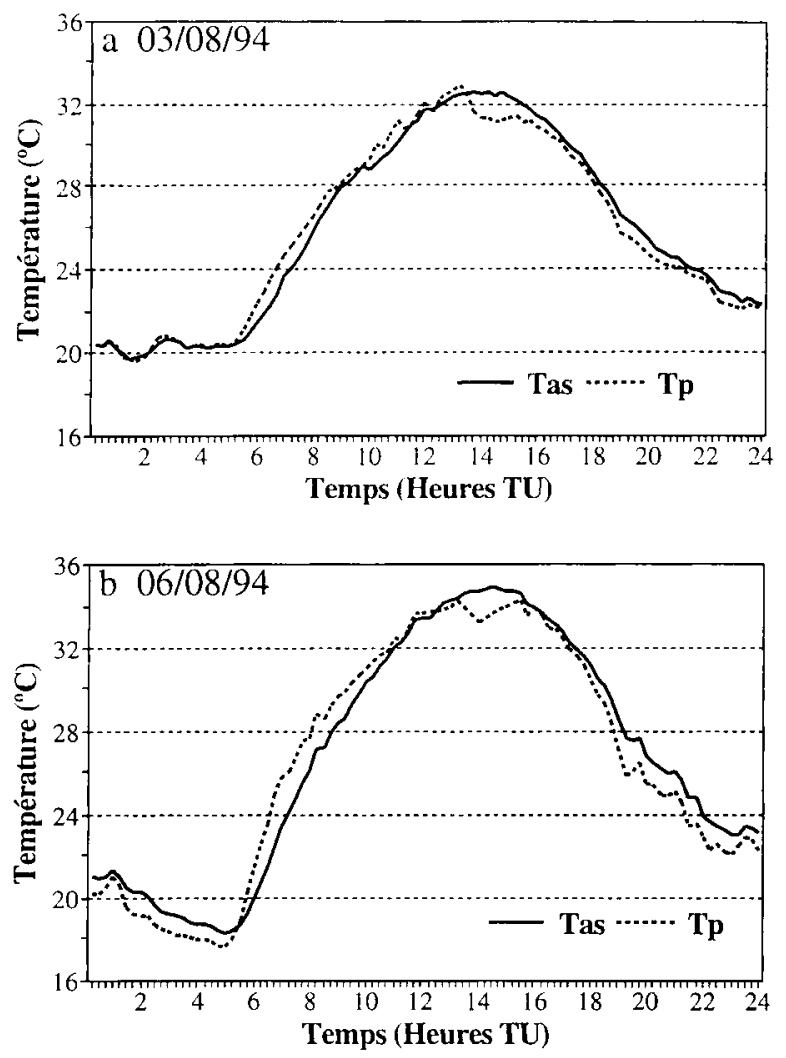

Fig 3. Comparaison entre l'évolution de la température de l'air au sommet du couvert $(1,7 \mathrm{~m})$ (Tas) et de la température des panicules (Tp) sur le site non irrigué $(H)$ le 3 août 1994 (a), le 6 août 1994 (b). 


\section{Modification de la température des organes reproducteurs et de la température de l'air par irrigation par aspersion}

À la différence du traitement irrigué hebdomadairement $(H)$ qui sert de témoin, le traitement irrigué quotidiennement $(\mathrm{J})$ pendant les heures chaudes de la journée était soumis à une irrigation intermittente commençant à 11 ou 12 heures et finissant à 17 heures TU, en utilisant une fréquence d'irrigation définie chaque jour. Les figures 4 et 5 montrent l'évolution de la température des organes reproducteurs au cours de l'irrigation du traitement $(\mathrm{J})$ comparativement au traitement $(\mathrm{H})$. On remarque que les évolutions de températures des épis ou des panicules sur les deux sites avant l'irrigation sont très proches, ce qui nous permet de justifier l'utilisation du traitement $(H)$ comme témoin microclimatique en l'absence de l'irrigation. Après le début de l'irrigation du site $(\mathrm{J})$, la réduction de la température des épis et des panicules sur le site $(\mathrm{J})$ est immédiate en raison de la perte d'énergie par évaporation à la surface des organes de l'eau dont le renouvellement est permanent du fait de l'irrigation par aspersion. Cet abaissement de la température des organes se maintient jsuqu'à l'arrêt de l'irrigation mais il n'est pas constant du fait de l'intermittence de l'irrigation ; l'abaissement maximal de la température coïncide généralement avec le maximum de la température des organes du traitement non irrigué $(H)$.

En comparant les graphiques (c) des figures 4 et 5 (jour 06/08/1994), on remarque que pour une même intensité et une même fréquence d'irrigation, le refroidissement des épis est inférieur à celui des panicules. Pour les épis, l'écart entre les deux sites est en moyenne de $3^{\circ} \mathrm{C}$ avec un maximum de $4^{\circ} \mathrm{C}$ alors que, pour les panicules, il est de $5^{\circ} \mathrm{C}$ en moyenne et atteint un maximum de $8^{\circ} \mathrm{C}$. Cela est dû aux différences de morphologie, de structure et de localisation entre les deux organes. Par ailleurs, l'épi reçoit probablement moins d'eau d'irrigation que la panicule car une partie de l'eau est interceptée par la strate foliaire située entre les organes mâles et femelles.

Fig 4. Évolution de la température des épis Te sur le site témoin $H$ (ligne continue) et sur le site irrigué quotidiennement $\mathrm{J}$ (pointillés) le 27 juillet $1994(3,8 \mathrm{~mm}$ d'irrigation, 2 minutes d'irrigation toutes les 5 minutes, intensité d'irrigation de 4,3 mm/heure) (a), le 3 août 1994 (1,8 $\mathrm{mm}$ d'irrigation, 2 minutes d'irrigation toutes les 20 minutes intensité d'irrigation de $7,6 \mathrm{~mm} /$ heure) (b), le 6 août $1994(6,8$ $\mathrm{mm}$ d'irrigation, 2 minutes d'irrigation toutes les 5 minutes, intensité d'irrigation de 7,6 mm/heure) (c).
La figure 6 montre les modifications de la température de l'air mesurée à trois niveaux du couvert. L'effet de l'irrigation sur la température de l'air n'est perceptible que 2 heures environ après le début de l'irrigation. Cette observation est valable pour toutes les journées étudiées quelle que soit la fréquence ou l'intensité d'irrigation utilisée et pour les trois niveaux de mesure. La majeure partie de l'effet de l'irrigation sur la température de l'air a lieu à l'intérieur du couvert (1 m) par évaporation de l'eau des surfaces
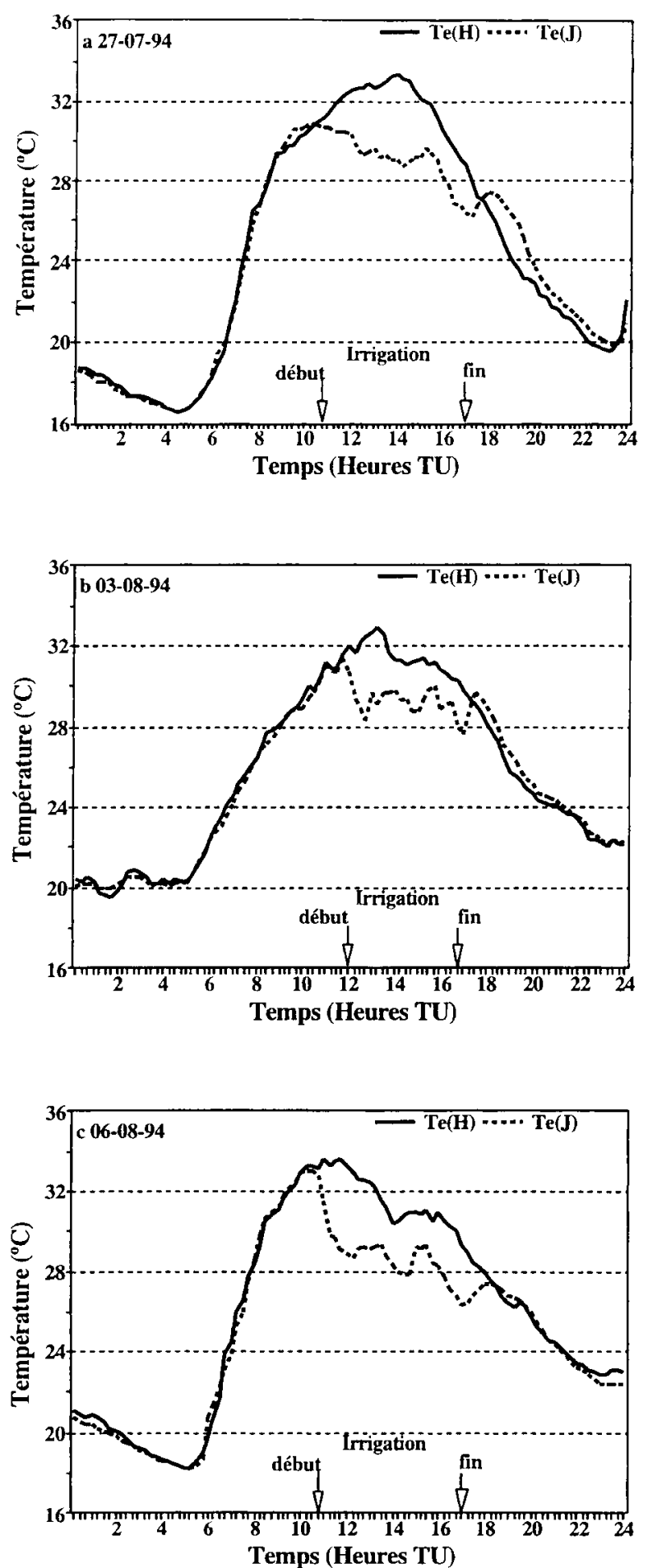

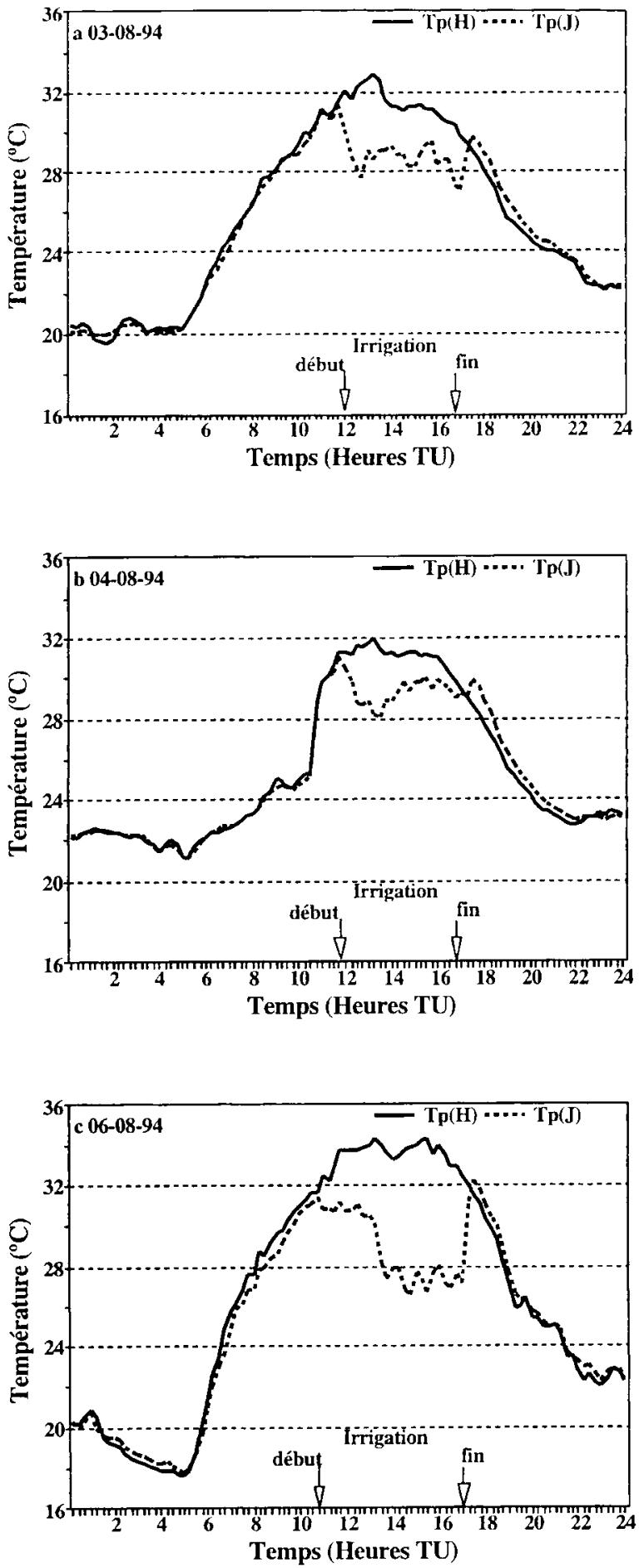

Fig 5. Évolution de la température des panicules Tp sur le site témoin $\mathrm{H}$ (ligne continue) et sur le site irrigué quotidiennement $\mathrm{J}$ (pointillés) le 3 août 1994 (1,8 m d'irrigaton, 2 minutes d'irrigation toutes les 20 minutes, intensité d'irrigation de $7,6 \mathrm{~mm} /$ heures) (a), le 4 août 1994 ( $2 \mathrm{~mm}$ d'irrigation, 2 minutes d'irrigation toutes les 10 minutes, intensité d'irrigation de $7,6 \mathrm{~mm} /$ heure) (b), le 6 août 1994 (6,8 mm d'irrigation), 2 minutes d'irrigation toutes les 5 minutes, intensité d'irrigation de 7,6 mm/heure) (c).
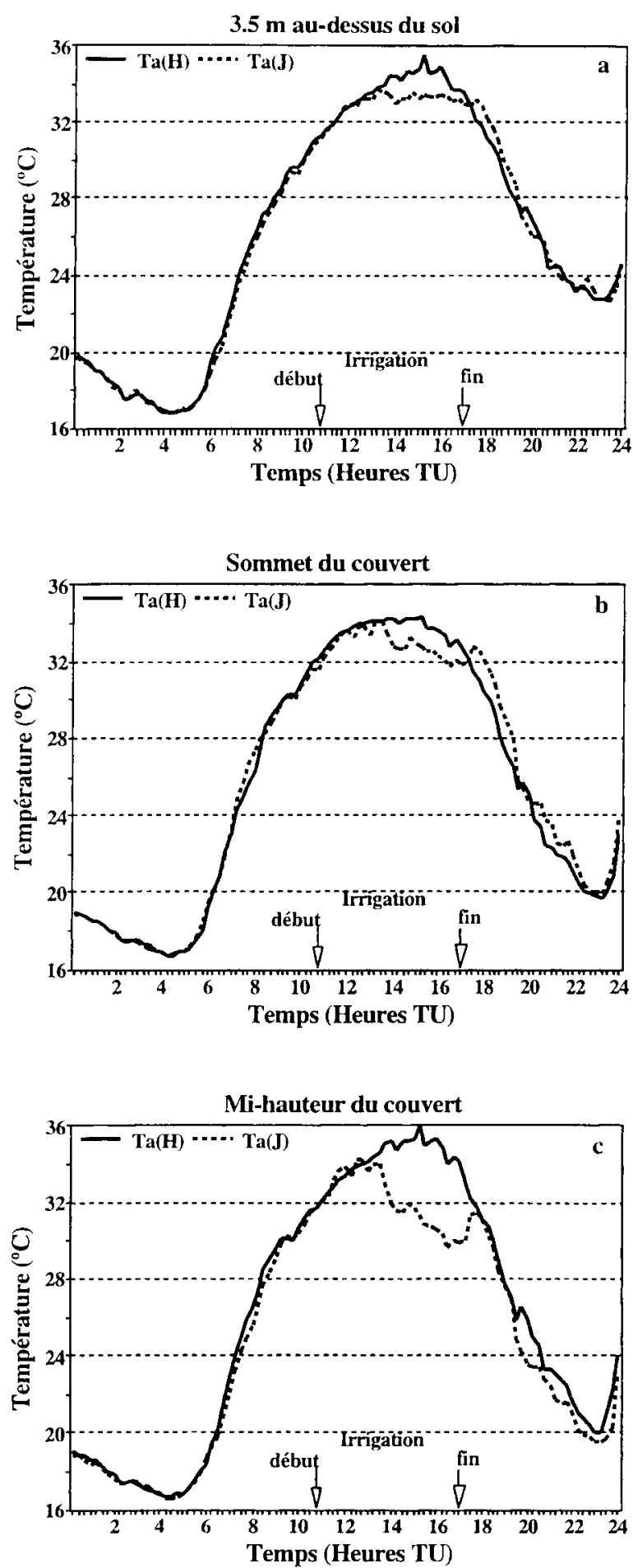

Fig 6. Évolution de la température de l'air Ta mesurée à trois niveaux : à 1,8 $\mathrm{m}$ au-dessus du couvert ( $3,5 \mathrm{~m}$ au-dessus du sol) (a), au sommet du couvert $(1,7 \mathrm{~m})$ (b) et à mi-hauteur du couvert $(1 \mathrm{~m})$ (c) sur le site témoin $\mathrm{H}$ (ligne continue) et le site irrigué quotidiennement $J$ (pointillés) le 27 juillet 1994 (3,8 $\mathrm{mm}$ d'irrigation et une fréquence de 2 minutes d'arrosage toutes les 5 minutes et une intensité de $4,3 \mathrm{~mm} /$ heure). 
mouillées (feuillages et sol), les écarts de température obtenus entre les deux traitements sont relativement élevés (jusqu'à $4{ }^{\circ} \mathrm{C}$ ) ; en revanche, l'abaissement de la température de l'air par irrigation apparaît nettement plus faible au sommet du couvert $(1,7 \mathrm{~m})$ et à $1,8 \mathrm{~m}$ au-dessus du couvert (3,5 $\mathrm{m}$ au-dessus du sol).

\section{Influence de l'intensité et de la fréquence d'irrigation sur le refroidissement}

Les écarts obtenus entre les deux traitements sont représentés sur la figure 7 qui met en relation les effets de l'intensité et de la fréquence d'irrigation sur le refroidissement des épis et l'air à trois niveaux du couvert. Sur cette figure, on remarque que, pour la température des épis (fig $7 a$ ), les deux intensités utilisées (4,3 et 7,6
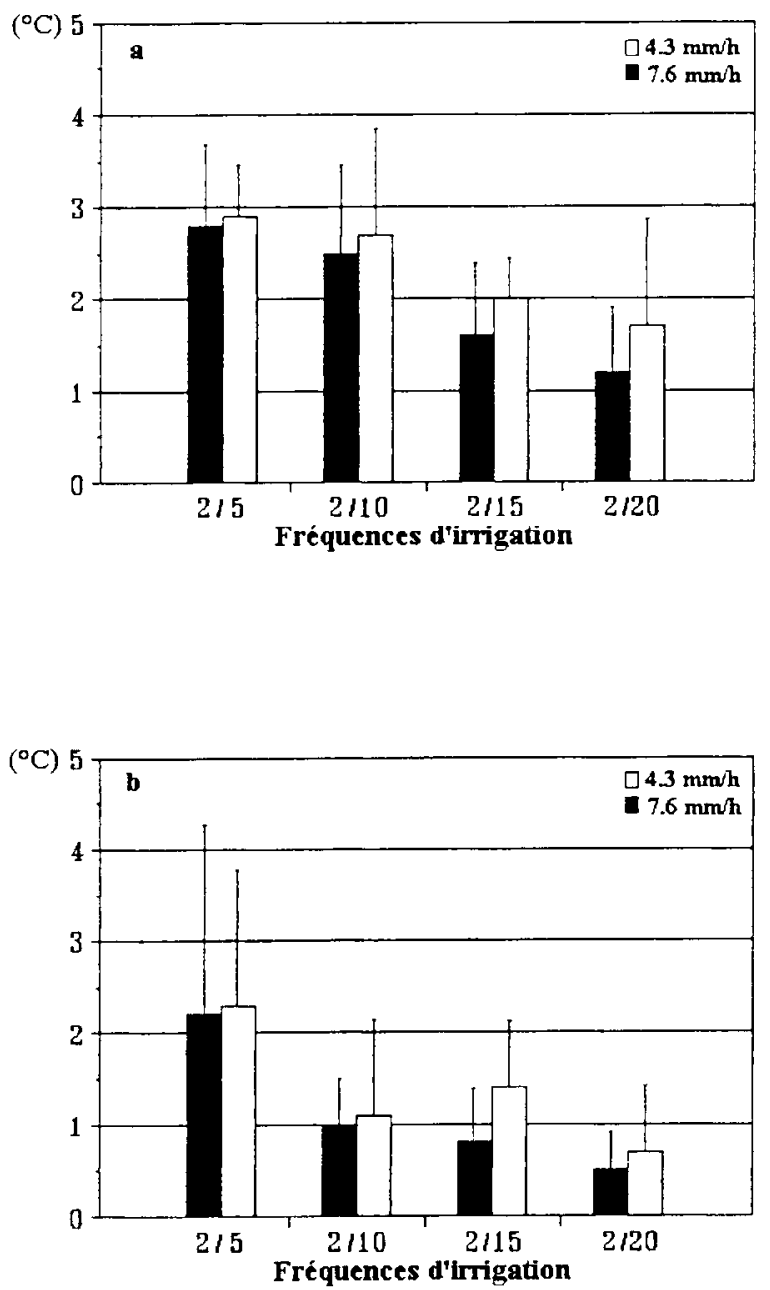

$\mathrm{mm} /$ heure) produisent le même refroidissement pour une fréquence d'irrigation donnée (la différence ne dépasse pas $0,5^{\circ} \mathrm{C}$ ). Les fréquences testées peuvent être regroupées en deux catégories : fortes fréquences (2 minutes d'arrosage toutes les 5 et 10 minutes) et faibles fréquences ( 2 minutes d'arrosage toutes les 15 et 20 minutes). Pour la première catégorie, l'écart moyen de la température des épis entre les deux traitements atteint $3^{\circ} \mathrm{C}$ avec un maximum autour de $4{ }^{\circ} \mathrm{C}$, alors que, pour la deuxième catégorie, l'écart est de $2^{\circ} \mathrm{C}$ avec un maximum de $3^{\circ} \mathrm{C}$.

Pour la température de l'air à mi-hauteur du couvert (fig $7 \mathrm{~b}$ ), la fréquence d'arrosage la plus forte ( 2 minutes arrosage toute les 5 minutes) détermine l'effet le plus net, l'écart atteint $4,5^{\circ} \mathrm{C}$ au maximum avec une moyenne de $2{ }^{\circ} \mathrm{C}$. Pour les autres fréquences ( 2 minutes d'arrosage toutes les 10,15 et 20 minutes), l'écart moyen
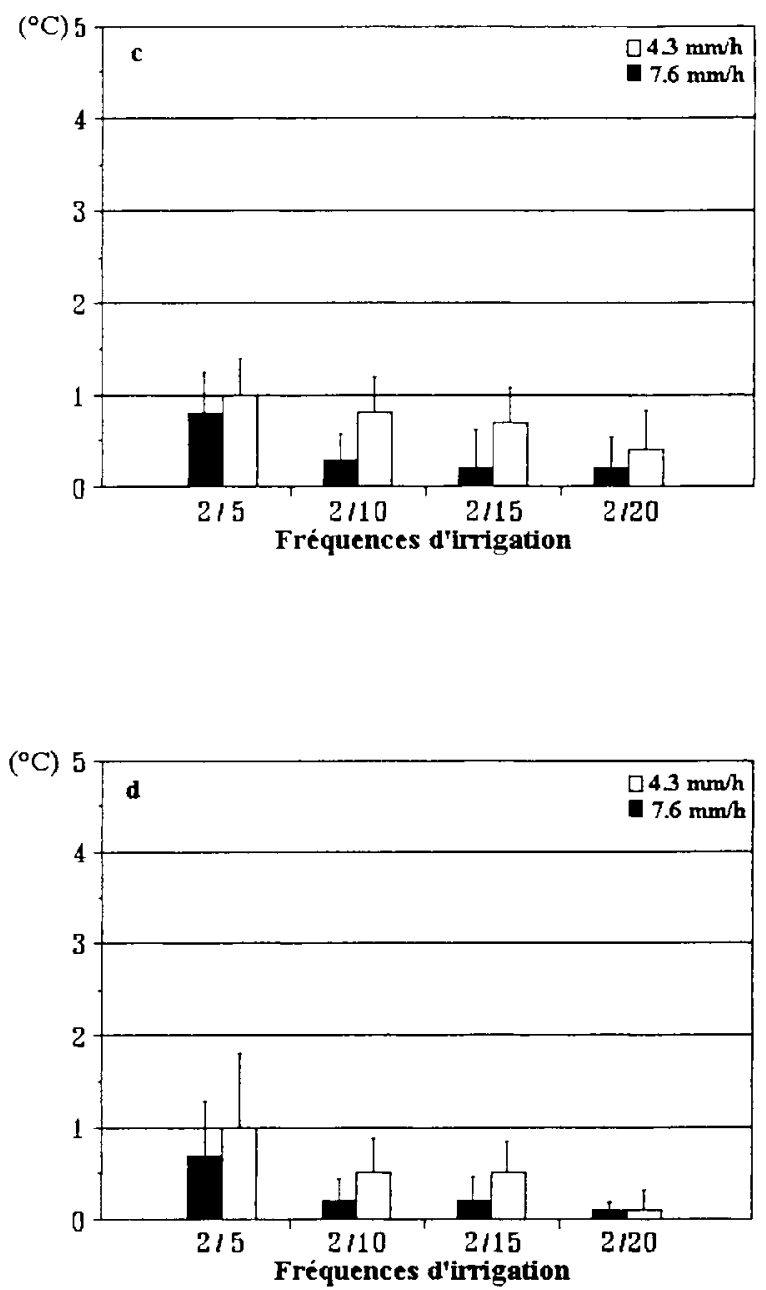

Fig 7. Différence moyenne de température entre la parcelle irriguée hebdomadairement $(\mathrm{H})$ et la parcelle irriguée quotidiennement $(\mathrm{J})$ pour les épis (a) et pour l'air mesurée à trois niveaux du couvert : à mi-hauteur du couvert (1 m) (b), au sommet du couvert (1,7 m) (c), à $3,5 \mathrm{~m}$ au-dessus du sol (1,8 $\mathrm{m}$ au-dessus du couvert) (d) en fonction de la fréquence d'irrigation (2 minutes d'arrosage toutes les 5 minutes, 2 minutes d'arrosage toutes les 10 minutes, 2 minutes d'arrosage toutes les 15 minutes et 2 minutes d'arrosage toutes les 20 minutes) et de l'intensité d'irrigation ( $: 4,3 \mathrm{~mm} /$ heure et $\downarrow 7,6 \mathrm{~mm} / \mathrm{heure}$ ). Les barres représentent l'écart type. 
oscille autour de $1^{\circ} \mathrm{C}$ et l'écart maximum varie en fonction de l'intensité (l'intensité la plus forte provoque le refroidissement le plus élevé) et de la fréquence d'irrigation utilisées.

L'irrigation provoque de très faibles modifications de la température de l'air mesurée au sommet du couvert et à 3,5 m au-dessus du sol (fig $7 \mathrm{c}$ et d) ; en effet, le maximum de réduction de la température ne dépasse pas $1,5^{\circ} \mathrm{C}$.

Des expérimentations similaires sur un couvert de maïs ont été menées aux États-Unis en climat semi-aride au Nebraska d'une part (Thompson et al, 1986) et au Kansas d'autre part (Steiner et al, 1983). Un point commun entre nos résultats et les résultats obtenus par ces auteurs est que la température des organes (épis, panicules, feuilles) diminue aussitôt après le début de l'irrigation. La différence d'amplitude du refroidissement dépend de la morphologie de l'organe végétal et du type d'irrigation utilisée. En conditions de demande climatique moins forte et au moyen d'une irrigation par pivot central, l'écart de température des feuilles obtenu par Steiner et al (1983) entre le couvert de maïs non irrigué et le couvert en cours d'irrigation est supérieur à ce que nous avons obtenu sur les épis par aspersion intermittente. En dehors des différences entre études dues d'une part aux caractéristiques climatiques durant l'irrigation et d'autre part à l'intensité d'irrigation utilisée (tableau II), le début de l'abaissement de la température de l'air après le début de l'irrigation est très dépendant de l'inten- sité et de la durée d'irrigation. Avec l'intensité utilisée dans le travail de Thompson et al (1986) $(11,6 \mathrm{~mm} /$ heure $)$, la réduction de la température de l'air intervient 10 minutes après le début de l'irrigation. Steiner et al (1983) ont obtenu une diminution de la température de l'air 1 heure après le début de l'irrigation en utilisant une intensité de $10 \mathrm{~mm} /$ heure. Dans notre étude, avec une intensité d'irrigation de $7,6 \mathrm{~mm} /$ heure et une irrigation intermittente, l'abaissement de la température de l'air est intervenu 2 heures après le début de l'irrigation ; cela tend à conforter le fait que plus l'intensité d'irrigation est forte et plus le délai entre le début de l'irrigation et le début de l'abaissement de la température est court.

Pour intervenir pendant des journées à haut risque de stress thermique, il est nécessaire de connaître les besoins de réduction des températures des organes végétaux et par conséquent la quantité d'eau d'irrigation requise pour ce refroidissement. Dans les conditions rencontrées, notre expérimentation montre qu'il existe une relation entre le taux de refroidissement et la hauteur d'eau apportée par irrigation. La figure 8 illustre, pour différentes journées climatiquement similaires pendant les heures chaudes de la journée, la relation entre la somme des écarts thermiques horaires entre les deux parcelles irriguée ( $\mathrm{J}$ ) et non irriguée $(\mathrm{H})$ et la quantité d'eau d'irrigation appliquée. Les écarts thermiques considérés sont relatifs à la température des épis, de l'air à mi-hauteur et au sommet du couvert et sont cal-

Tableau II. Comparaison de résultats obtenus dans le cadre de cette étude (le 6 août 1994) avec ceux obtenus par Steiner et al (1983) et Thompson et al (1986) sur le refroidissement des organes végétaux par aspersion.

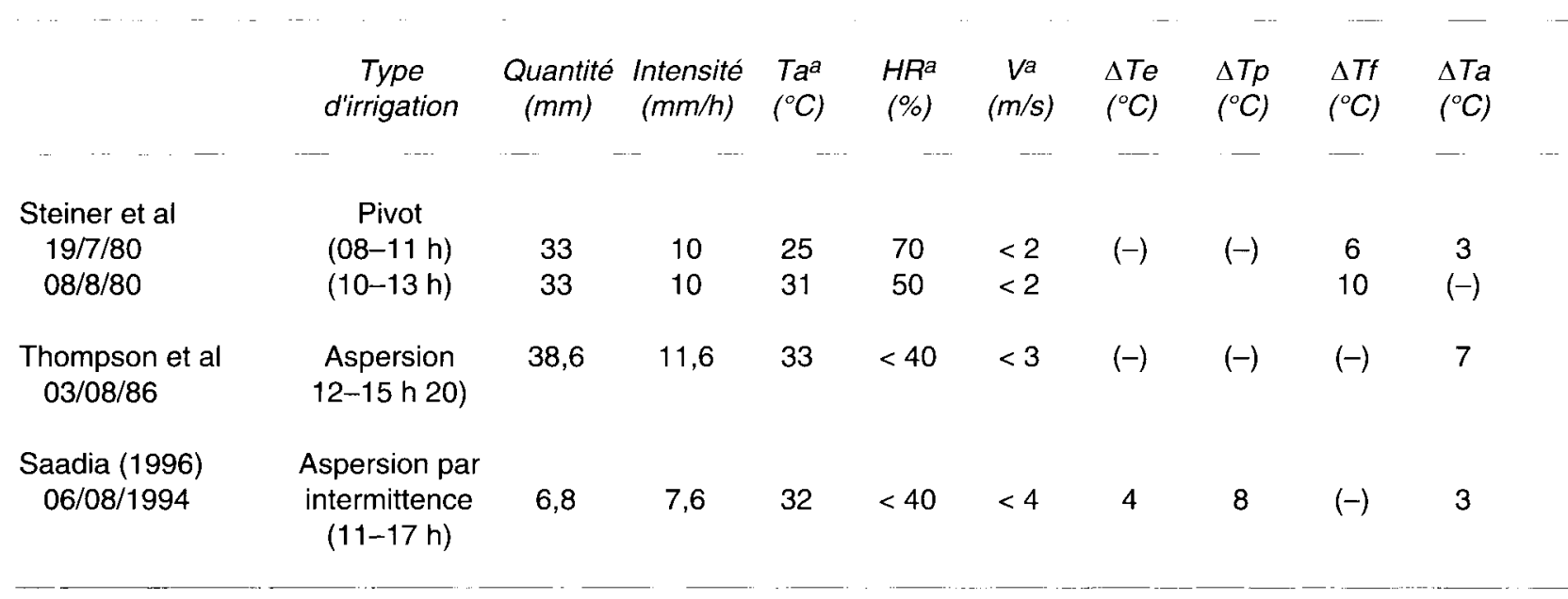

Ta : température de l'air, HR : humidité relative de l'air, $V:$ vitese du vent, $\Delta T e$ : réduction maximale de la température des épis, $\Delta T p$ : réduction maximale de la température des panicules, $\Delta \mathrm{Tf}:$ réduction maximale de la température des feuilles, $\Delta \mathrm{Ta}:$ réduction maximale de la température de l'air au sommet du couvert. a Paramètres mesurés au début de l'irrigation ; (-) valeurs non mesurées. 
culés après 2 et 5 heures depuis le début de l'irrigation. Dans des conditions climatiques et d'irrigation similaires, une telle relation permet de quantifier en première approximation la quantité d'eau nécessaire pour un besoin de refroidissement connu.

\section{Réduction de la durée d'occurrence des fortes températures}

Un stress thermique se caractérise par une durée d'occurrence et une intensité variable en fonction de l'écart entre la température appliquée et la température optimale pour la croissance de la plante (Levitt, 1980 ; Schöffl et al, 1988). Pour la gestion des stress thermiques, il est important de caractériser ces deux composantes du stress et en particulier de quantifier l'influence de l'irrigation sur la réduction de la durée d'occurrence de températures supérieures à un seuil donné.

Pour mettre en évidence l'effet de l'irrigation sur la réduction de la durée du stress thermique, trois journées ont été sélectionnées le 27 juillet, le 8 août et le 19 août pour la température des épis et les 3,4 et 8 août pour la température des panicules. Ces journées sont caractérisées par des températures élevées (supérieures à $30^{\circ} \mathrm{C}$ entre 11 et 12 heures). La durée relative (D) de dépassement d'un seuil thermique ( $T$ ) donné par la température réelle des organes reproducteurs est exprimée en pourcentage de la durée d'irrigation (de 11 à 17 heures TU dans notre expérimentation).

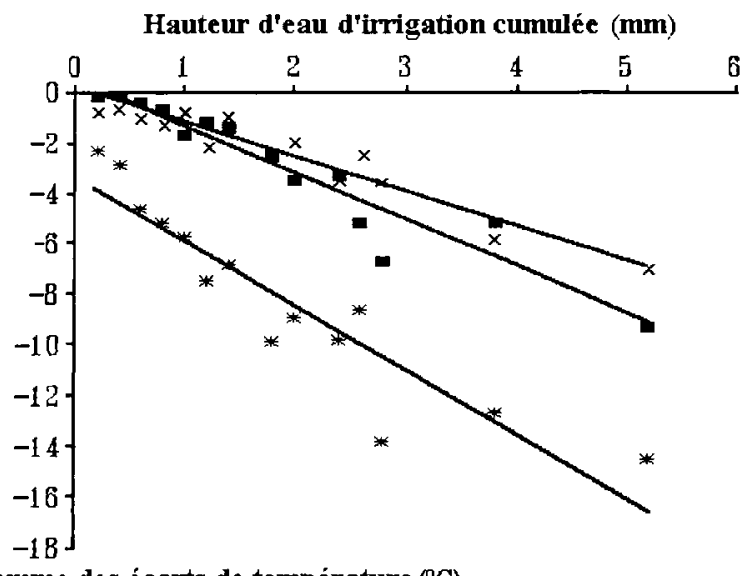

Somme des écarts de température $\left({ }^{\circ} \mathrm{C}\right)$

Fig 8. Relation entre la somme (calculée sur une base horaire) des différences de température des épis (Te), et de température de l'air mesurée à trois niveaux de couvert : à mi-hauteur du couvert (Tai), au sommet du couvert (Tas) entre la parcelle irriguée hebdomadairement $(H)$ et la parcelle irriguée quotidiennement $(\mathrm{J})$ en fonction de la quantité d'eau apportée, $\left(^{*}\right)$ Tp : $\left(r^{2}=0,85\right)(\square)$ Tai $:\left(r^{2}=0,91\right),(x)$ Tas $:\left(r^{2}\right.$ $=0,92)$.
Si on considère des seuils arbitraires de température (situés entre 26 et $33^{\circ} \mathrm{C}$ ) susceptibles d'être atteints ou dépassés par la température des organes des deux parcelles $(H)$ et $(J)$, on remarque que la durée relative pendant laquelle la température des épis et panicules des deux traitements (fig 9) dépasse les seuils de 26, 27 et $28{ }^{\circ} \mathrm{C}$ est élevée sur la parcelle $(\mathrm{H})$ (entre 80 et $100 \%$ de la durée d'irrigation) ; dans cette gamme de seuils thermiques, l'intervention de l'irrigation n'affecte que faiblement cette durée sur la parcelle (J). La différence entre les deux traitements $(H)$ et $(J)$ devient très nette en raison de l'intervention de l'irrigation à partir du seuil de $29{ }^{\circ} \mathrm{C}$ où la durée de dépassement de ce seuil
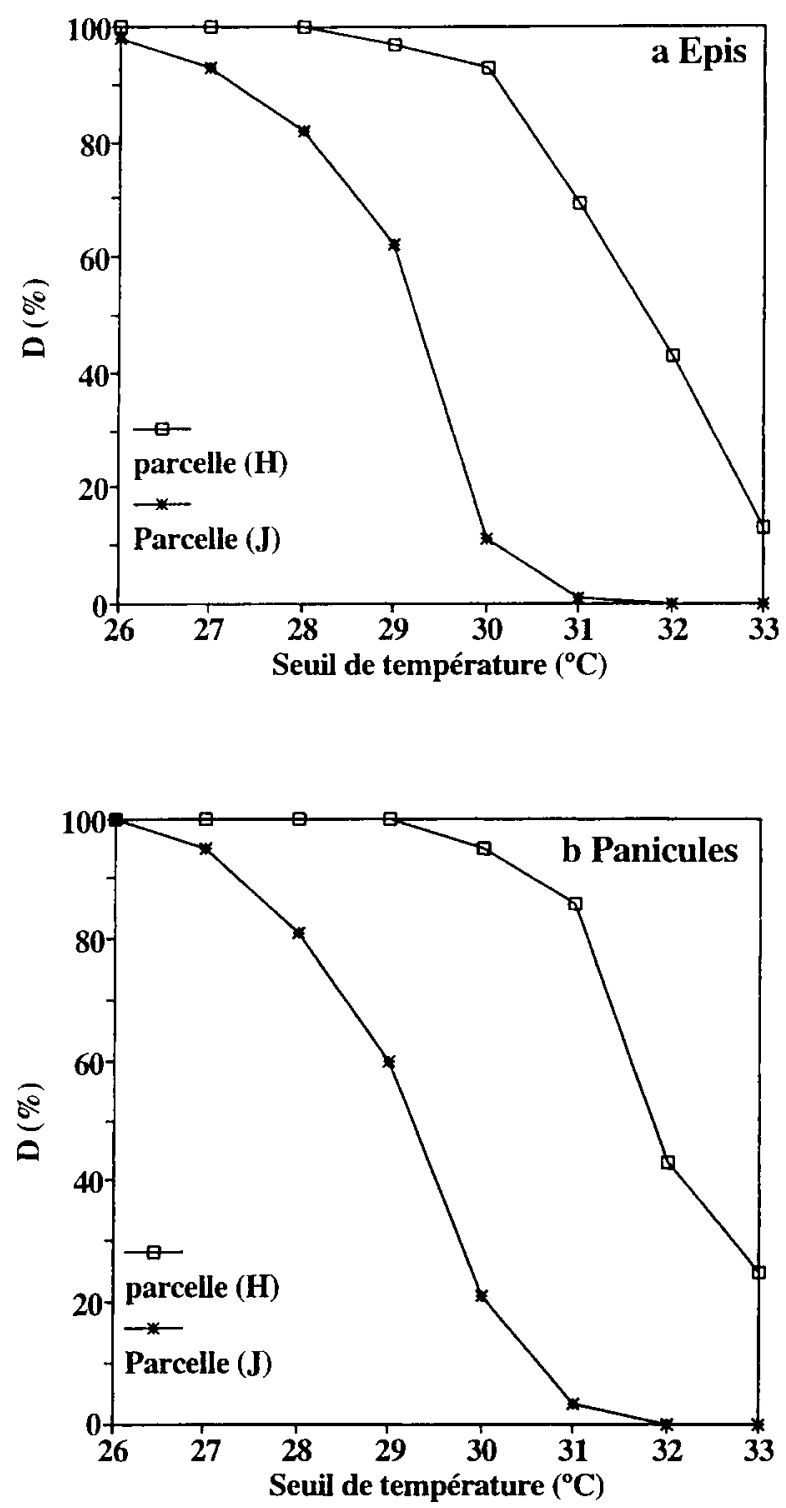

Fig 9. Comparaison entre les traitements $H$ et $J$ des durées relatives (D) pendant lesquelles la température des épis (a) ou des panicules (b) dépasse un seuil de température donné ; la durée relative (D) est exprimée en pourcentage de la durée d'irrigation (moyenne des 27 juillet, 8 août et 19 août pour la température des épis et des 3,4 et 8 août pour la température des panicules). 
sur le traitement irrigué $(\mathrm{J})$ est de $60 \%$ alors qu'elle est de $100 \%$ sur le traitement $(H)$. Pour les seuils 31,32 et $33^{\circ} \mathrm{C}$, cette durée est comprise entre 20 et $80 \%$ sur le traitement $(H)$; elle est réduite pratiquement à $0 \%$ sur le traitement en cours d'irrigation $(\mathrm{J})$. L'analyse de la durée d'occurrence des fortes températures confirme le rôle de l'irrigation par aspersion qui contribue à la continuité de l'humectation des organes reproducteurs ce qui est une condition de la permanence du refroidissement évaporatif. Dans cette formulation de la durée d'occurrence de fortes températures, aucune hypothèse n'est faite sur la relation stress-durée ; dans une approche qui irait plus loin que la simple considération d'un seuil thermique, il serait intéressant de prendre en compte l'intensité du stress en fonction de l'écart entre la température optimale et la température appliquée.

\section{CONCLUSION}

Les conditions climatiques rencontrées pendant la période expérimentale furent très favorables pour tester l'irrigation comme outil de refroidissement par évaporation (température de l'air et rayonnement élevés, humidité de l'air et vitesse du vent faibles). L'influence de l'irrigation s'est traduite par la diminution de la température des organes reproducteurs et de l'air à l'intérieur du couvert. Le refroidissement des panicules est plus important que celui des épis, ce qui constitue un avantage pour la méthode sachant que le pollen est très vulnérable au stress thermique qui peut aboutir à la perte du pouvoir fécondant et de la viabilité de celui-ci.

La modification de la température des épis et de l'air induite par l'irrigation est beaucoup plus liée à la fréquence des irrigations qu'à l'intensité de celle-ci. En effet, la fréquence élevée (2 minutes d'arrosage toutes les 5 minutes) détermine un plus fort refroidissement que la fréquence faible ( 2 minutes d'arrosage toutes les 10 minutes), tandis qu'il existe très peu de différence entre les abaissements de température observés pour des intensités de $4,3 \mathrm{~mm} /$ heure et $7,6 \mathrm{~mm} /$ heure.

L'irrigation par aspersion permet de réduire la température des organes reproducteurs du maïs, ce qui conduit à la réduction de l'intensité d'un stress thermique éventuel ; par ailleurs, la durée d'occurrence de fortes températures est elle aussi fortement réduite. La mise en jeu du mécanisme de refroidissement par évaporation ne nécessite pas l'utilisation d'intensités d'irrigation élevées, ce qui permet une utilisation de la méthode sans incidence majeure en termes de consommation d'eau pendant les périodes de forte demande climatique.

L'intérêt de l'irrigation par aspersion en couverture intégrale en tant qu'outil de réduction des risques de stress thermique est confirmé par cette expérimentation. Pour l'application pratique, il convient de connaître les conséquences mécaniques éventuelles de l'aspersion juste après l'émission du pollen hors des anthères ; en effet, l'irrigation pourrait provoquer un lessivage des grains de pollen ; toutefois, le pollen est généralement émis le matin à partir de 10 heures et commence à germer dès son dépôt sur les soies. Par ailleurs, l'humectation du couvert végétal due à l'irrigation pourrait être à l'origine de contaminations fongiques ; cette éventualité est peu vraisemblable sachant que la protection fongicide du maïs est bien maîtrisée et que l'humectation du couvert végétal s'interrompt peu de temps après l'arrêt de l'aspersion en raison de la forte demande évaporative, ce qui conduit à de faibles risques de contamination.

La faisabilité technique de cette méthode doit être évaluée en situation réelle de production de semences ; l'intérêt économique reste également à évaluer sous deux aspects : i) la vérification du maintien d'une bonne fécondation en appliquant cette méthode en situation de stress thermique ; ii) l'évaluation du risque climatique de stress thermique.

\section{RÉFÉRENCES}

Binelli G, Vieria E, Ottaviano E (1985) Temperature effects on pollen germination and pollen tube growth in maize. Genet Agrar 39, 269-281

Dupuis I, Dumas C (1990) Influence of temperature stress on in vitro fertilization and heat stock proteins synthesis in maize productive tissues. J Plant Physiol 94, 665-670

Forva C, Binelli G, Ottaviano E (1986) Male gametophyte response to high temperature in maize. In : Biotechnology of Pollen (DL Mulcahy, GB Mulcahy, E Ottaviano, eds), Springer-Verlag, New York, États-Unis, 33-38

Gagliardi D (1994) Expression et régulation des gènes HSP au cours du développement du pollen de maïs. Thèse doctorat biochimie et biologie moléculaire, Lyon, France, 1-144

Griffin RE, Richardson EA (1979) Sprinklers for microclimate cooling of bud development. In : Modification of the Aerial Environment of Plants (BJ 
Barfield, JF Gerber, eds), Am Soc Agric Engr, Saint Josef, MI, États-Unis

Herrero MP, Johnson RR (1980) High temperature stress and pollen viability of maize. Crop Sci 20 , 796-800

Jones RJ, Gegenbach BG, Cardwel VB (1981) Temperature effects on in vitro kernel development of maize. Crop Sci 21, 761-766

Kohl RA, Wright JL (1974) Air temperature and vapour pressure changes caused by sprinkler irrigation. Agron J 66, 85-88

Lavergne P (1993) Microclimat du champ de maïs semence et fécondation. Incidence de l'irrigation. Mémoire de fin d'études ESAP, Toulouse, $92 \mathrm{p}$.

Levitt J (1980) Responses of Plants to Environmental Stresses: Chilling, Freezing, and High Temperature Stresses, Vol I, 2nd edition. Academic Press Inc, New York, États-Unis

Lomas J, Mandel M (1973) The quantitative effects of two methods of sprinkler irrigation on the microclimate of a mature avocado plantation. Agric Meteorol 12, 35-48

Mitchell JC, Petolino JF (1988) Heat stress effects on isolated reproductive organs of maize. J Plant Physiol 133, 625-628

Moss GI, Downey LA (1971) Influence of drought stress on female gametophyte development in corn subsequent grain yield. Crop Sci 11, 368-372

Saadia R (1996) Modification du microclimat d'un couvert de maïs au moyen de l'irrigation par aspersion en vue de la gestion des stress thermiques. Thèse doctorat, Ensa, Rennes, $80 \mathrm{p}$
Schöffl F, Baumann G, Raschke E (1988) The expression of heat shock genes. A model for environmental response. In : Plant Gene Research. Temporal and Spatial Regulation of Plant Genes (DPS Verma, RB Goldberg, eds), Springer-Verlag, New York, États-Unis, 253-273

Schoper JB, Lambert RJ, Vasilas BL (1986) Maize pollen viability and ear receptivity under water an high temperature stress. Crop Sci 26, 1029-1033

Schoper JB, Lambert RJ, Vasilas BL (1987b) Pollen viability, pollen shedding and combining ability for tassel heat tolerance in maize. Crop Sci 27, 27-31

Seginer I (1970) A resistance model of evaporation during sprinkling. Agric Meteorol 7, 487-497

Steiner JL, Kanemasu ET, Hasza D (1983) Microclimatic and crop responses to center pivot sprinkler and to surface irrigation. Irrig Sci 4, 801814

Thompson AL, Gilley JR, Norman JM (1986) Simulation of sprinkler water droplet evaporation above a plant canopy. ASAE Paper No 86-2108, Saint Joseph, MI, États-Unis

Thompson AL, Gilley JR, Norman JM (1993) A sprinkler water droplet evaporation and plant canopy model. II. Model application. Transactions of ASAE 36(3), 743-750

Westgates ME, Boyer JS (1986) Reproduction at low silk and pollen potentials in maize. Crop Sci 26, 951-956

Wright JL, Stevens JL, Brown MJ (1981) Controlled cooling of onion umbels by periodic sprinkling. Agron J 73, 481-490 